\title{
¿ESTÁ EL CLIMA MEDITERRÁNEO SUJETO A CICLOS?: INTERACCIONES ENERGÉTICAS ENTRE LA TEMPERATURA DEL ATLÁNTICO NORTE Y LA CICLOGÉNESIS MEDITERRÁNEA
}

\author{
José QUEREDA SALA, Enrique MONTON CHIVA \\ y Victoria QUEREDA VÁZQUEZ \\ Laboratorio de Climatología. Instituto Interuniversitario de Geografia. Universidad Jaume I. \\ Avda. Sos Baynat s/n, 12071 Castelló de La Plana. \\ quereda@uji.es, montone@uji.es, mquereda@uji.es
}

\section{RESUMEN}

Este estudio tiene por objetivo esencial el responder a una de las más inquietantes cuestiones que actualmente se plantean a la Climatología mediterránea: ¿Está nuestro clima regional sujeto a ciclos?. Un objetivo básico que se fundamenta, inicialmente, en el análisis de las teleconexiones energéticas observadas entre el campo térmico del Atlántico N (SST y observatorios subpolares) y la ciclogénesis de la cuenca occidental del Mediterráneo. Ciclogénesis activada mediante el drenaje del aire frío polar sobre las cálidas aguas de la cuenca occidental del Mediterráneo. Una cuenca donde la resonancia del NAOi, ha sido puesta en cuestión. Ciertamente, la configuración orográfica en cubeta, donde los sistemas atlánticos experimentan una fuerte afección de momento angular sobre las montañas circundantes (Egger and Hoinka, 2008) y un fondo marino extraordinariamente convectivo, determinan una zona muy autárquica. A pesar de ello resulta difícil aceptar que el campo térmico del Atlántico Norte, como reflejo del estado energético hemisférico, carezca de influencia en el drenaje de aire polar sobre las aguas cálidas del Mediterráneo activador de la ciclogénesis convectiva. En este orden de conocimientos la presente investigación muestra que los gradientes del campo térmico en el Atlántico Norte juegan un importante papel en la activación de la ciclogénesis mediterránea. Simultáneamente el calor latente englobado en esa ciclogénesis mediterránea parece inyectarse en la circulación superior para restablecer el equilibrio energético.

Palabras claves: Interacciones energéticas, balance térmico hemisférico, ciclogénesis mediterránea, calor latente, Oscilación del Atlántico Norte.

\begin{abstract}
The objective of this study is essentially to respond to one of the most troubling questions currently being asked of Mediterranean Climatology: Is our regional climate subject to cycles? An authentic research challenge given the predictions of the latest Report, Fifth Assessment (IPCC WGI AR5, 2013-2014), on the highly vulnerable water resources in our Mediterranean region. This basic objective is based initially on the analysis of the teleconnections observed between the North Atlantic thermal field (SST, sea surface temperature, and subpolar observatories) and the cylclogenesis of the Western Mediterranean basin. A cyclogenesis activated by the draining of cold polar air over the warm waters of the Western Mediterranean basin, where the resonance
\end{abstract}


of the NAOi (the pressure index in the North Atlantic), has been brought into question. Indeed, the basin orographic configuration, where the Mediterranean systems have for now a strong angular momentum transfer over the surrounding mountains (Egger and Hoinka, 2008) and an extraordinarily convective sea bed, define what is a very autarkic zone. Despite that, it is difficult to accept that the North Atlantic thermal field, as a reflection of the hemispheric energy state, does not influence the drainage of polar air over the warm waters of the Mediterranean to activate the convective cyclogenesis. In this regard, this work shows that the surface thermal gradients of the North Atlantic seem to be the trigger of the Mediterranean cyclogenetic activity through the draining of polar cold air over the warm Mediterranean waters. Simultaneously, the latent heat included in Mediterranean cyclogenesis is injected and diffused in high circulation to restore the hemispheric thermal balance.

Key words: energy interactions, hemispheric thermal balance, Mediterranean cyclogenesis, latent heat, North Atlantic Oscillation.

\section{INTRODUCCIÓN E INTERÉS}

El presente estudio, como culminación de una extensa trayectoria de investigaciones sobre la climatología mediterránea, trata de responder a una de las más inquietantes cuestiones que actualmente se plantean a la Climatología mediterránea: ¿Está nuestro clima regional sujeto a ciclos? Un auténtico reto investigador que actualmente adquiere una gran trascendencia a tenor de las predicciones que los modelos de cambio climático establecen sobre la región mediterránea. En este orden de conocimientos, el Fourth Assessment del IPCC (Panel Intergubernamental para el Cambio Climático), Climate Change, 2007, de las Naciones Unidas y la presentación de las bases científicas del último lnforme, Fifth Assessment (IPCC WGI AR5, 2013-2014), basados en los modelos del Hadley Centre, establecen que nuestra región mediterránea será la más vulnerable de Europa a los efectos del cambio climático. Vulnerabilidad debida a que, simultáneamente al aumento térmico regional y de las evapotranspiraciones, se produciría una reducción de recursos hídricos, como consecuencia básica de un proceso atmosférico. Este proceso sería el desplazamiento hacia el norte de la zona neurálgica de formación de borrascas entre el fluido atmosférico polar y el tropical como consecuencia de la contracción del vortex polar (Fig. 1).

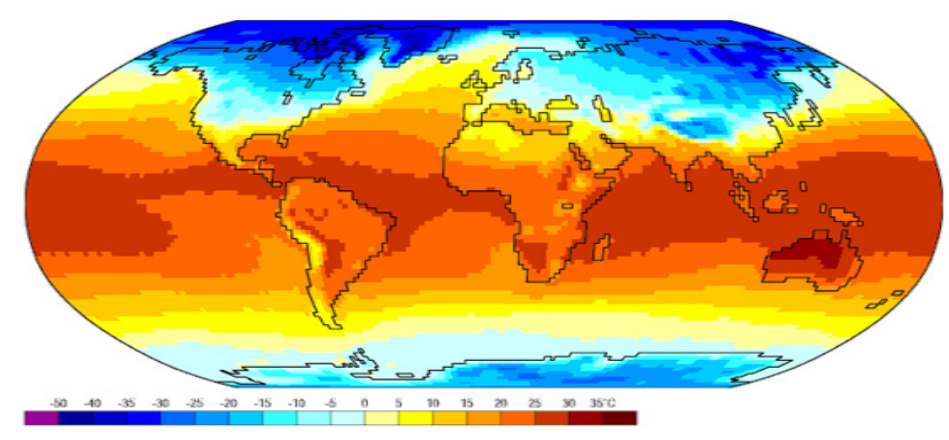

Fig. 1. Estado medio de los fluidos atmosféricos durante el mes de diciembre. La contracción latitudinal del fluido polar suscita las mayores alertas sobre la posible desertificación mediterránea. 
En efecto, en los modelos basados en las interacciones atmósfera-océano sobre el funcionamiento del sistema climático, el desplazamiento hacia el Norte de la zona subtropical boreal de altas presiones reducirá considerablemente la extensión de la zona de lluvias invernales, ahora responsable de la alimentación en agua de la región mediterránea (Flohn, 1979; Quereda et al., 1996). Como consecuencia de ello sobre la región Mediterránea se produciría una marcada disminución de la lluvia del orden de un 25 a un $30 \%$ en el transcurso de los próximos 40-50 años (Parry et al., 1999 y Quereda et al., 2000). Este es el modelo dinámico que sigue figurando en las bases científicas de los dos últimos informes del IPCC (2007 y 2013) (Fig. 2).

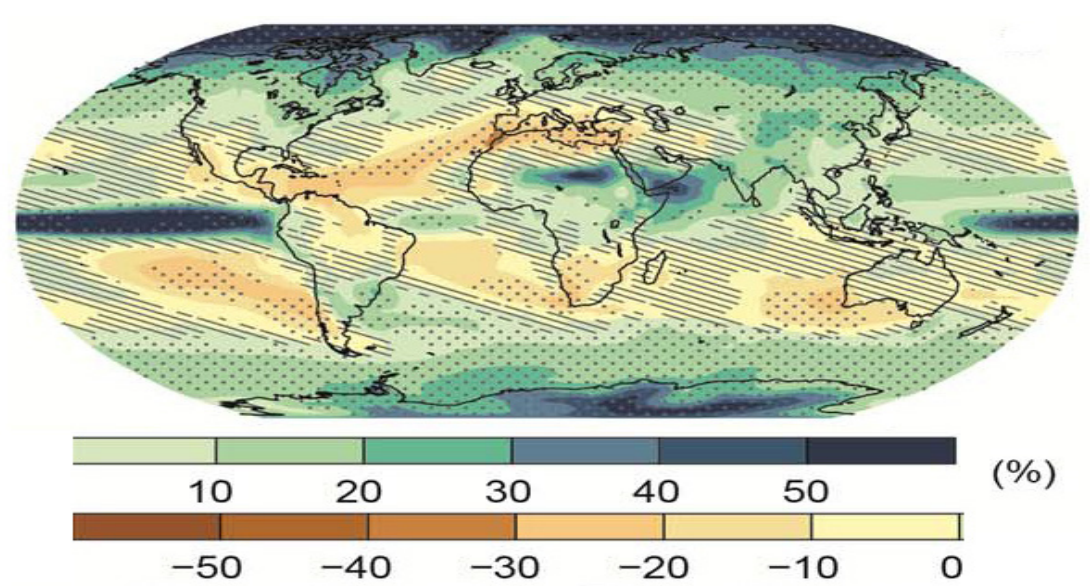

Fig. 2. Reducción de la escorrentía en el horizonte de los años 2080-2100 (Fifth Assessment, IPCC, 2013).

\section{OBJETIVO BÁSICO Y FUNDAMENTOS CIENTÍFICOS}

De este modo, la presente investigación tiene por objetivo esencial el responder a una de las más inquietantes cuestiones que actualmente se plantean a la Climatología mediterránea: ¿Está nuestro clima regional sujeto a ciclos?. Este objetivo básico se fundamenta inicialmente en el análisis de las teleconexiones energéticas observadas entre el campo térmico del Atlántico N (SST, sea surface temperature, y observatorios subpolares) y la ciclogénesis de la cuenca occidental del Mediterráneo. Ciclogénesis activada mediante el drenaje del aire frío polar sobre las cálidas aguas mediterráneas. Simultáneamente, el calor latente englobado en las ciclogénesis mediterráneas es inyectado y difundido en la circulación de altura para restablecer el equilibrio térmico hemisférico.

El balance energético hemisférico juega un importante papel en la generación de los patrones de la circulación atmosférica. Estos patrones de la CGA (Circulación General de la Atmósfera) aparecen muy vinculados a las interacciones atmósfera-océano (Watanabe and Kimoto, 2006). Este forzamiento atmosférico (Rodwell and Folland, 2002), es el desarrollado en el modelo cerrado atmósfera-océano Had-CM3 del Hadley Center (IPCC). Las circulaciones meridianas, con intensificación de baroclinidad y ondas ciclogenéticas muestran una gran sensibilidad con las fases negativas de la 
NAO (Feldstein, 2007; Courtenay and Davis, 2007). De modo contrario, las anomalías positivas de la SST sobre latitudes de $40^{\circ} \mathrm{N}$ parecen excitar la fase positiva de la $\mathrm{NAO}$, con reducción de ondas ciclogenéticas y acumulación de aire frio en latitudes septentrionales. Ello comporta igualmente una reducción de la intensidad y frecuencia de las situaciones de bloqueo con promedio de seis días frente a los once días de la NAO negativa (Buech and Nakamura, 2007).

En este orden de conocimientos se inscribe el objetivo de nuestro trabajo sobre la cuenca occidental del Mediterráneo, donde la resonancia del NAOi (índice de presión en el Atlántico Norte), ha sido puesta en cuestión (Martin Vide y López Bustins, 2006). Ciertamente, la configuración orográfica en cubeta, donde los sistemas atlánticos experimentan una fuerte afección de momento angular sobre las montañas circundantes (Egger and Hoinka, 2008) y un fondo marino extraordinariamente convectivo, determinan una zona muy autárquica. A pesar de ello resulta difícil aceptar que el campo térmico del Atlántico Norte, como reflejo del estado energético hemisférico, carezca de influencia en el drenaje de aire polar sobre las aguas cálidas del Mediterráneo para activar la ciclogénesis convectiva.

De este modo el objetivo principal del presente proyecto es el de analizar las posibles resonancias ciclogenéticas de la atmósfera mediterránea al forzamiento energético del estado térmico en el Atlántico Norte. En este orden de conocimientos, proponemos una simple teoría que explora las bases físicas de esta resonancia como un reajuste energético hemisférico. Proceso que se corresponde, en gran parte, con el transporte de calor englobado en las ciclogénesis de la cuenca occidental del Mediterráneo.

\section{METODOLOGÍA Y TÉCNICAS}

El análisis de las interacciones atmósfera-mar como proceso clave de la climatología mediterránea ha permitido establecer las bases de la teoría formulada. Un análisis que ha estado fundamentado sobre el conocimiento de la formación del campo térmico superficial en la cuenca occidental del Mediterráneo mediante los registros del observatorio universitario marino sobre la plataforma de BPOil (39 $56^{\circ} 42^{\prime}$ ' $\mathrm{N}$ y $00^{\circ} 01$ '36”E) (Figura 3). Este observatorio está excepcionalmente situado sobre la plataforma petrolífera de BPOil (300 m de longitud y orientación E-W), a $3 \mathrm{Km}$ de la costa, 12 metros de altura sobre el nivel del mar y sobre isobata de $-20 \mathrm{~m}$. Las coordenadas geográficas son $39^{\circ} 56^{\prime} 42^{\prime \prime} \mathrm{N}$ y $00^{\circ} 01^{\prime} 36^{\prime \prime} \mathrm{E}$. En funcionamiento convencional desde 1981, el observatorio ha sido completamente automatizado desde mediados del año 2000 mediante dos estaciones simultáneas CASELLA-DAVIS. Estas estaciones están compuestas de todo el equipamiento de sensores meteorológicos además de las tres sondas termométricas de Temperatura superficial del mar(TSM) al nivel de -1 y $-3 \mathrm{~m}$ y a $-7 \mathrm{~m}$. Unos registros cuya publicación y análisis permite a los investigadores de la Universitat Jaume I reiterar su enorme gratitud a BPOil.

El acusado contraste estacional del campo térmico se revela clave para unos procesos convectivos muy determinantes de la ciclogénesis mediterránea y de su originalidad. Procesos que, al mismo tiempo, han mostrado su vinculación a la misma dinámica del sistema climático, regido por la necesidad de transferencia térmica meridiana. 


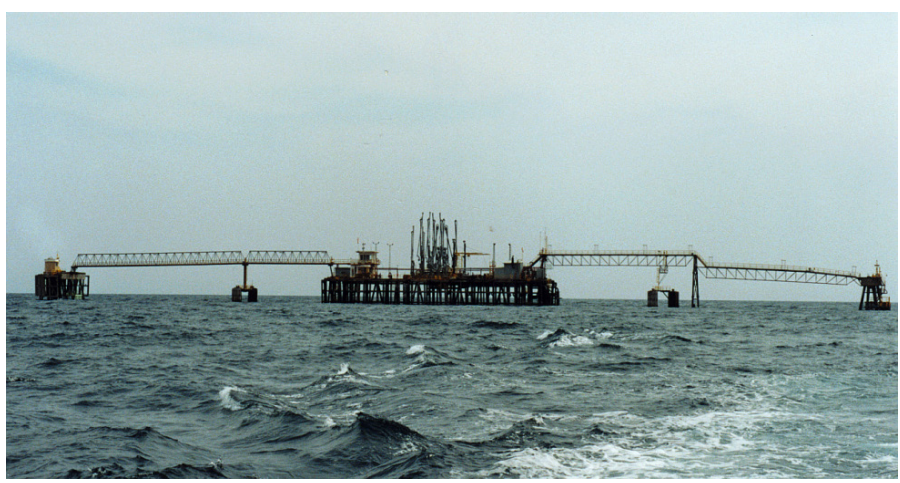

Fig. 3. Observatorio marino de la Universitat Jaume I, en el extremo izquierdo de la plataforma de BPOil, sobre isóbata de -20 metros, (3956'42 "N y $00^{\circ} 01$ '36"E).

\subsection{El campo térmico marino}

La cuenca occidental del Mediterráneo constituye una zona neurálgica por lo que concierne a las interacciones atmósfera-mar. Tanto las largas situaciones de estabilidad y sequía como, especialmente, la génesis y evolución de sus grandes perturbaciones meteorológicas vienen determinadas por el desarrollo más o menos intenso de los procesos convectivos que se generan en el interior de esa cubeta marina. Esta singularidad, ya puesta de manifiesto por Jansá $(1951,1966)$ y reconocida en el Coloquio de Roma sobre la Meteorología de la Cuenca Mediterránea (1962), es consecuencia del destacado papel que, en su génesis, juegan los factores geográficos. Dentro de esos factores debe considerarse muy especialmente la configuración del campo térmico marino (Figura 4).

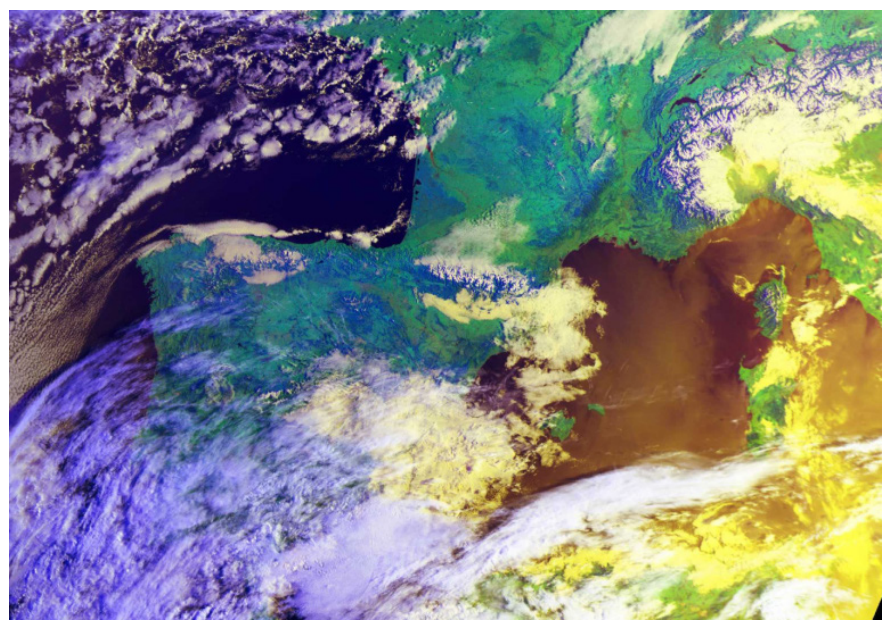

Fig.4. La cuenca del Mediterráneo occidental se configura como una cubeta de alto reborde

donde las capas de aire no son fáciles de renovar. El aerosol africano, apreciable en la imagen, es exponente de la naturaleza tropical continental de la masa de aire mediterránea (Imagen NOAA-18, 29 de abril de 2016, canal visible, Universidad Jaume I). 
Desde el fondo de esa cubeta, por sus cálidas temperaturas, su gran calor específico, convectividad y potencial evaporativo, la masa marítima es clave en los procesos climáticos (Noussier et al., 2008). Así y como inicial exponente de ello, la línea de costa representa una fuerte discontinuidad térmica. Es suficiente a este respecto comparar la temperatura media anual del aire en Castellón, $17^{\circ} \mathrm{C}$, con el estado térmico medio de las aguas a 2,5 $\mathrm{Km}$ de su línea de costa, $19^{\circ} \mathrm{C}$. Este gradiente térmico tierra-mar viene potenciado además por el existente entre la superficie marina y la capa de aire situada sobre ella a 10 metros de altura, $17,9^{\circ} \mathrm{C}$. Ambos contrastes térmicos, horizontal y vertical, son mucho más acusados desde finales de verano hasta principios de invierno (Figura 5). Así, la temperatura media de la superficie marina entre octubre y diciembre es de $17,9^{\circ} \mathrm{C}$ mientras que la de la capa de aire situada sobre ella es de $15,4^{\circ} \mathrm{C}$. Este gradiente es ya un factor de convectividad potencial cuya exageración está presente en la mayor parte de las perturbaciones atmosféricas mediterráneas.

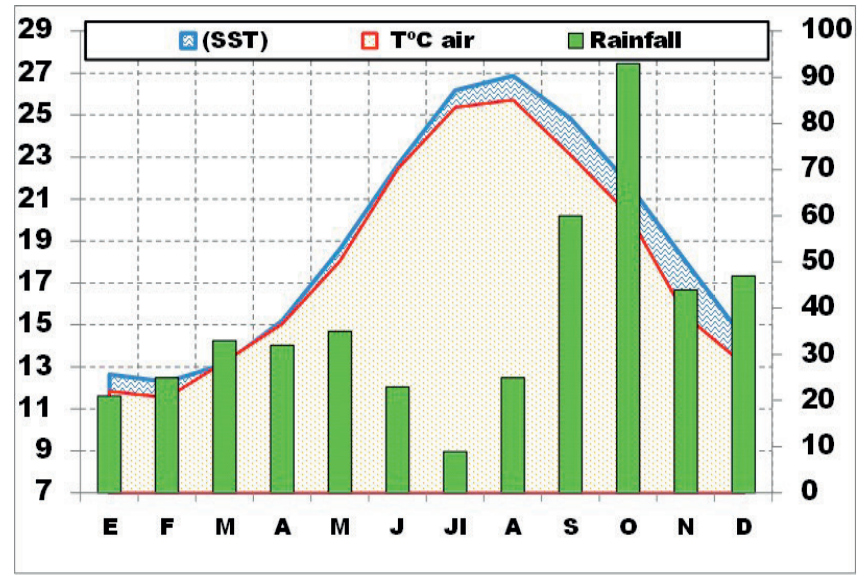

Fig. 5. Regímenes térmicos (2001-2014), del aire y de la SST, (Observatorio marino de la Universidad Jaume I). El gradiente térmico aparece como un potencial factor de convectividad que suele estar presente durante las perturbaciones atmosféricas mediterráneas.

Este campo térmico va a ser decisivo para unos intercambios energéticos, atmósfera-mar, claves en la climatología mediterránea. Así, durante el otoño e invierno la SST suele ser más elevada que la del aire. Ello engendra una activa emisión de calor hacia la atmósfera con enfriamiento de las aguas superficiales (Figura 6). La subsidencia de las aguas marinas contribuye a crear una profunda homotermia. La amplitud térmica media diaria invernal de la SST-TSM, temperatura superficial del mar, es de $0,32^{\circ} \mathrm{C}$, frente a los $0,47^{\circ} \mathrm{C}$ del verano.

Por el contrario, durante el verano, la intensa radiación solar y las altas temperaturas ambientales, bajo la persistente situación de estabilidad anticiclónica, limitan la turbulencia convectiva. La termoclina aparece muy en superficie y la capa activa implicada en los intercambios con la atmósfera se torna muy delgada. Consecuentemente, el calentamiento es más intenso en ese volumen más superficial del agua. En este orden de procesos, las observaciones actuales vienen detectando que durante la 
época estival es cuando se registra la máxima variabilidad en la formación de anomalías térmicas superficiales. Así, los análisis de las variaciones en las anomalías térmicas superficiales de los océanos Atlántico $\mathrm{N}$ y Pacífico $\mathrm{N}$ han mostrado que su máximo acontece en la estación cálida, entre julio y septiembre, al igual que en el Mediterráneo occidental donde la variación estival, hasta $3^{\circ} \mathrm{C}$, es el doble que la invernal (Quereda, 1986; Alekseev and Bagryantsev, 1987).

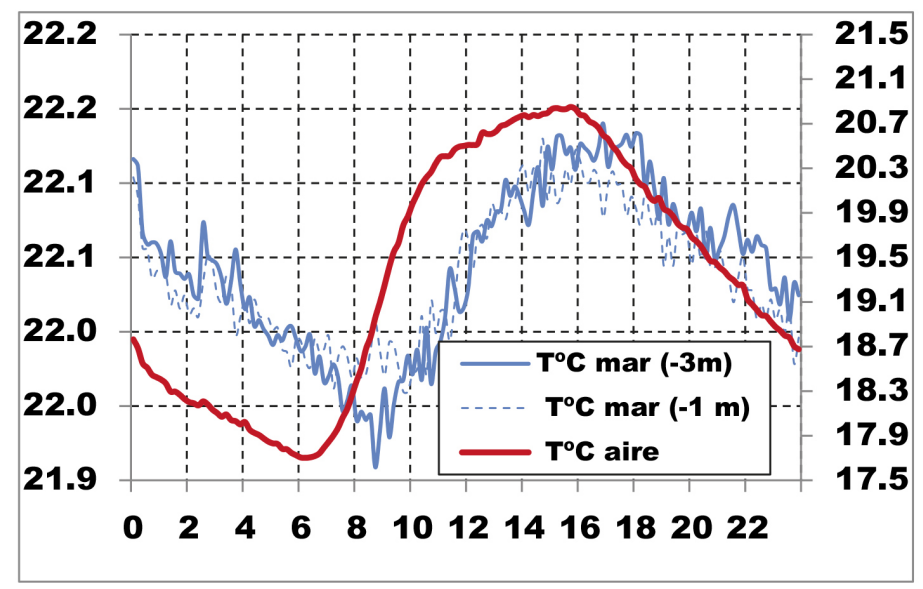

Fig. 6. El perfil térmico vertical del otoño (septiembre-noviembre) en régimen diario, muestra la constitución de una capa superficial de gran homotermia. El gradiente térmico medio diario entre la superficie y la isóbata de $-3 \mathrm{~m}$ es tan sólo de $0.2 / 0.4{ }^{\circ} \mathrm{C}$. Observatorio universitario marino (2005-2014).

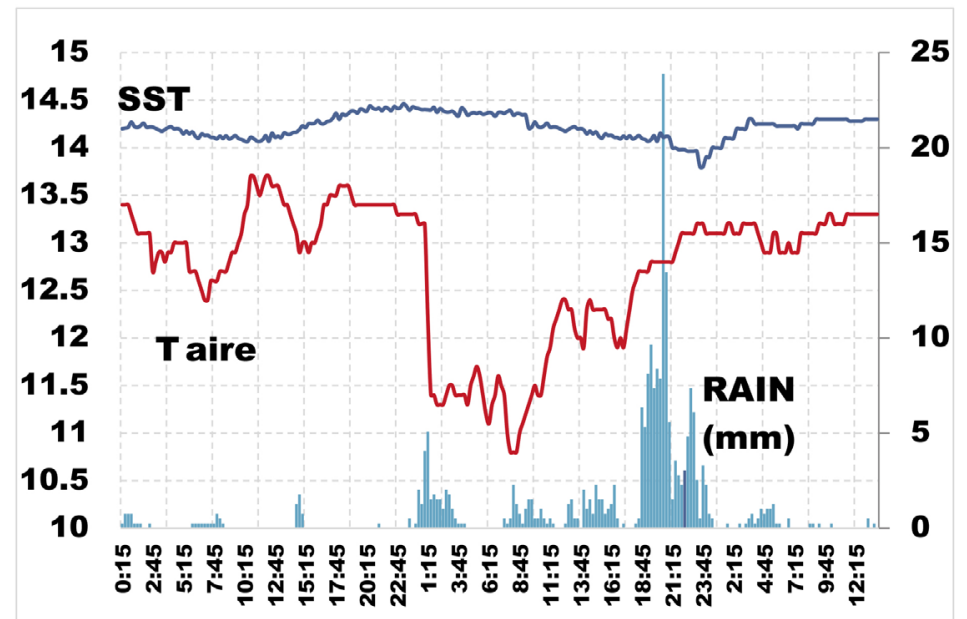

Fig. 7. Gráfico de las interacciones atmósfera-mar durante los días 21 a 23 de marzo de 2015. La gran ciclogénesis del día 22 (record histórico con $210 \mathrm{~mm}$ ), viene precedida por la exageración del gradiente entre la SST y la capa de aire suprayacente (Observatorio universitario marino). 
Las anomalías positivas acumuladas de la SST no sólo pueden ser coadyuvantes del mismo drenaje de aire polar hacia la cuenca occidental del Mediterráneo, sino que simultáneamente actúan como factor básico de las interacciones atmósfera-mar. La SST se erige así en superficie altamente convectiva con respecto a la capa de aire suprayacente. Estas mismas observaciones permiten situar un umbral o valor crítico de alta convección en valores de gradiente térmico aire-mar superiores a $4-5^{\circ} \mathrm{C}$. Una intensa evaporación precede a las grandes ciclogénesis. Las sondas térmicas marinas acusan un notable enfriamiento con hundimiento de las aguas superficiales y remonte de aguas más profundas (Figura 7).

\subsection{Los factores atmosféricos}

Aunque determinante, el efecto termoconvectivo del mar no lo es todo en la climatología regional. Es más, su verdadero papel lo ejerce mediante la interacción constante con la atmósfera suprayacente. Procesos cuya estacionalidad muestra su vinculación a la misma dinámica del sistema climático. Este sistema aparece regido por una necesidad básica de transferencia térmica meridiana (Vonder Haar and Oort, 1973). Tal es el teorema fundamental de la circulación descubierto por V. Bejerknes en 1897 y más recientemente formulado en función de la denominada oscilación del Atlántico Norte, NAO, (North Atlantic Oscillation) (Visbeck, 2001).

Esta necesidad de transferencia térmica meridiana, verdadero motor de la Circulación General de la Atmósfera (CGA), ha sido establecida mediante la variación del índice de ciclo, $\mathrm{I}(\lambda)$, a través de los registros de la velocidad del viento geostrófico así como del gradiente de la temperatura atmosférica $30-60^{\circ}$, a nivel de $700 \mathrm{hPa}$, sobre el Atlántico Norte. Un análisis que, en nuestro estudio, hemos basado en el promedio dinámico y térmico sobre un arco longitudinal $\left(12.5^{\circ} \mathrm{E}-17.5^{\circ} \mathrm{W}\right)$. Metodología que ofrece un valor más riguroso del cohesionado energético hemisférico con respecto a la utilizada en los índices NAOi, PNA (Pacific/North American) y otros, establecidos en dos o cuatro puntos de referencia. Unos índices que, caso del NAOi, quedarían en suspenso, ante la previsión de los modelos de cambio climático de una intensificación y desplazamiento hacia el NE de los dos grandes centros de presión del Atlántico $\mathrm{N}$ (Zeng-Zeng and Zhaohua, 2004). Todo ello sin menoscabo del amplio reconocimiento que los análisis actuales conceden al acoplamiento de los patrones de la CGA con las fases NAO sobre el Atlántico Norte (Gulev et al., 2000; Stephenson et al., 2000; Marshall et al., 2002; Trigo et al. 2004; Andersson et al., 2010).

Las investigaciones recientes han mostrado que las resonancias de la atmósfera a las anomalías de la SST ofrecen diversas respuestas a escala planetaria (Colin de Verdière and Blanc, 2001). Específicamente, en el Atlántico Norte, las correlaciones estacionales han mostrado una buena significación durante el semestre invernal (Quereda et al, 2011). Incluso estas teleconexiones entre la variabilidad invernal de la SST y el forzamiento atmosférico han sido verificadas en la Europa oriental a través del EAWR (East Atlantic-West Russia) (Kazmin et al., 2009). Unas resonancias que hemos tratado de verificar, sobre la cuenca occidental del Mediterráneo, mediante la comparación de los ciclos que muestran tanto SST como la temperatura en los principales observatorios subpolares del Atlántico Norte, con los de las precipitaciones mediterráneas. 
Estas resonancias son reflejo del valor de los intercambios térmicos entre el aite polar y el aire tropical. La aceptable correlación inversa obtenida $(-0,72)$ entre las precipitaciones mediterráneas y el gradiente térmico en las aguas superficiales del Atlántico Norte muestra el determinante papel de las interacciones energéticas entre la SST del Atlántico Norte y las precipitaciones mediterráneas. En nuestro análisis, SST es el promedio de los paralelos $37^{\circ} \mathrm{N}$ y $70^{\circ} \mathrm{N}$, sobre las coordenadas longitudinales del arco $12,5^{\circ} \mathrm{E}-17,5^{\circ} \mathrm{W}$. Como hemos dicho, esta metodología proporciona un valor más representativo del estado energético hemisférico que el obtenido con los usos de los índices NAOi, PNA (Pacific-North American) y otros semejantes, basados tan sólo en unos pocos puntos de referencia (Figura 8).

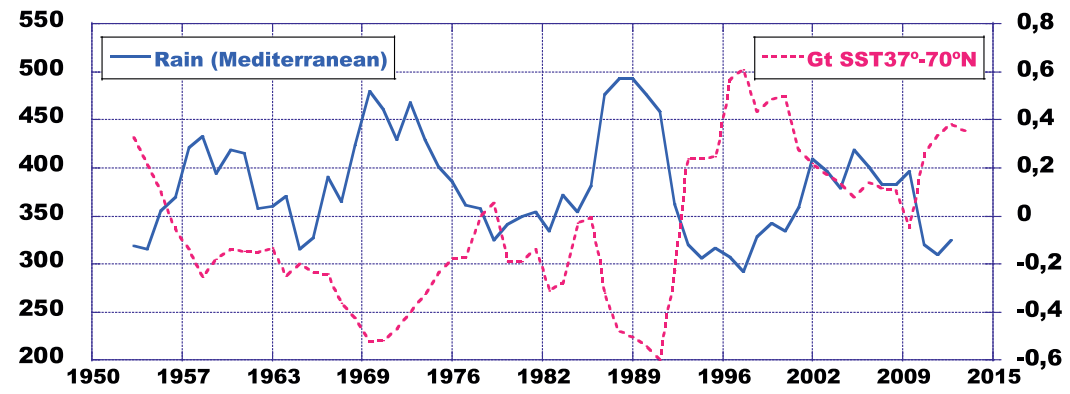

Fig. 8. La evolución de la SST en el Atlántico Norte y de las precipitaciones mediterráneas muestra la profunda interacción energética hemisférica. Evolución suavizada ( 3 años, linea intermitente en rojo) del gradiente térmico (Gt SST) $37^{\circ} \mathrm{N}-70^{\circ} \mathrm{N}$ (promedio $12.5^{\circ} \mathrm{E}$ a 17.5

${ }^{\circ} W$ ). Fuente: HadSST2 data set (Rayner et al., 2014). Las precipitaciones son promedio de los observatorios de primer orden de Murcia, Alicante, Valencia y Castellón, (AEMET).

\section{RESULTADOS: LAS TELECONEXIONES ENERGÉTICAS ENTRE LA ACTIVIDAD CICLOGENÉTICA MEDITERRÁNEA Y LA SST DEL ATLÁNTICO NORTE}

Las grandes anomalías de la circulación general tales como las ciclogénesis explosivas son consecuencia de anomalías semejantes en la distribución de la energía (Namias, 1987 y 1989). Ello sugiere que las grandes anomalías en el campo térmico del Atlántico Norte podrían ser una de tales alertas (Quereda, 1994, 1989 a y 1989 b, 2011). En este orden de procesos es lícito plantearse la hipótesis de que las anomalías energéticas detectadas por la SST pudiesen estar en la base de los ciclos pluviométricos mediterráneos. De este modo, cuando la acumulación de aire frío en las latitudes polares o de aire cálido en la zona tropical o en la misma cuenca mediterránea son excesivas, la atmósfera se comporta como un gigantesco condensador que se descarga bien con erupciones de aire frío o bien con dorsales cálidas activadoras de los bloqueos. Son precisamente éstos los mecanismos que, durante las situaciones con índice de circulación zonal bajo, provocan la alteración de las típicas situaciones de estabilidad por las situaciones de inestabilidad y precipitaciones sobre la fachada oriental de España. Este es el proceso que parecen mostrar las teleconexiones energéticas entre la actividad ciclogenética mediterránea y la temperatura regional del Atlántico N (Figura 9). 


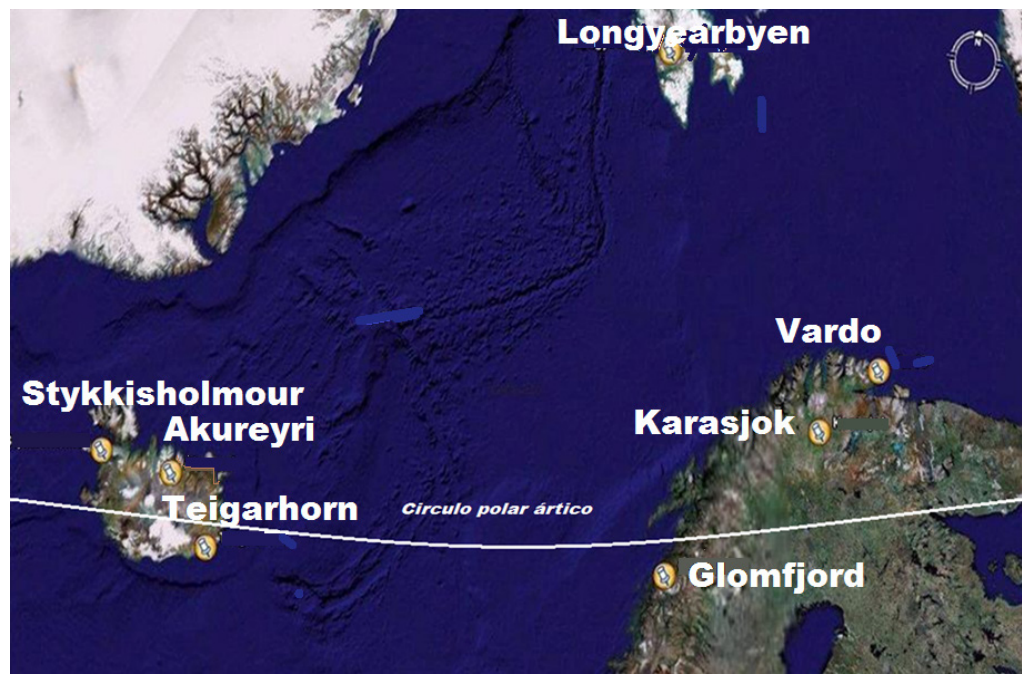

Fig. 9. Observatorios polares utilizados en la presente interacción energética como campo térmico del Atlántico Norte. Norwegian Meteorological Institute (MET) e Icelandic Met Office.

A través de estos procesos de descarga polar sobre la cuenca mediterránea, la energía sacada de los reservorios oceánicomarinos subtropicales es redistribuida atmosféricamente. Así, en la figura 10, con fuente en los registros de temperatura de los observatorios polares desde finales del siglo XIX, es posible observar como la difusión de calor hacia la troposfera polar se efectúa mediante el calor latente englobado en las ciclogénesis mediterráneas e inyectado en la circulación de altura. El test t-Student da un valor de correlación próxima a 0.6 y una probabilidad de correlación de $<0,0001$, indicativa de alta significación entre ambas variables. Incluso puede constatarse que unas temperaturas polares más elevadas, con disminución del gradiente térmico hemisférico, determinan ciclos pluviométricos con mayor severidad de sequía. Este parece ser el caso del ciclo más reciente

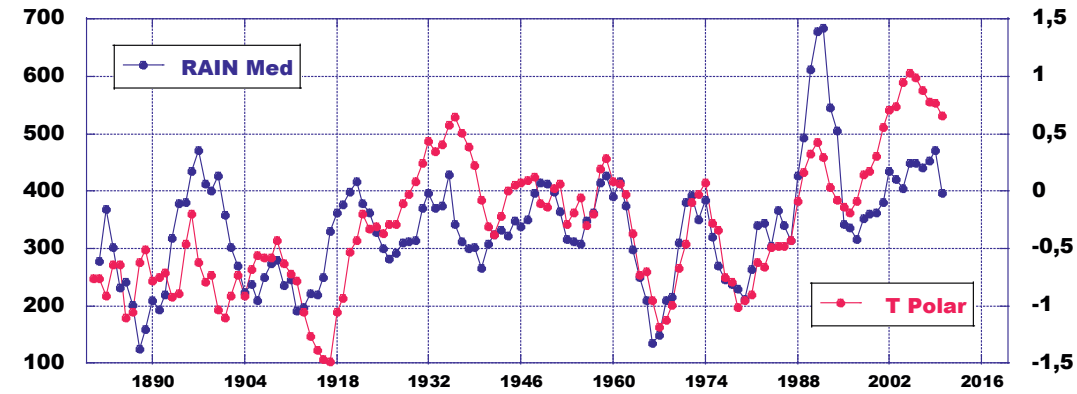

Fig. 10. Evolución térmica suavizada mediante medias móviles de 5 años (en rojo) de los observatorios subpolares (en Islandia, Akureyri, Teigarhorn y Stykkishólmur, en Noruega, Vardo, Karasjok, Glomfjord y Longyearbyen) y de las precipitaciones mediterráneas, con idéntica suavización, (en azul). Norwegian Meteorological Institute (MET), Icelandic Met Office y AEMET. 


\section{REFERENCIAS Y FUENTES}

Alekseev, G. V. and Bagryantsev, M. V. (1987). "The climate role of ocean surface temperature anomalier in the warm half of the year". Soviet Meteorology and Hidrology, 5, pp.52-56.

Andersson, A. Bakan, S. and Grabl, H. (2010). "Satellite derived precipitation and freshwater flux variability and its dependence of the North Atlantic Oscillation". Tellus, vol. 62, pp. 453-468.

Buech, Ch. and Nakamura, Hi. (2007). "Scandinavian pattern and its climatic impact". Quaterly Journal of Royal Meteorology Society, 133, pp. 2117-2131.

Colin de Verdière, A. and Blanc, M. L. (2001). "Thermal resonance of the atmosphere to SST anomalies. Implications for the Antartic circumpolar wawe". Tellus, vol.53, pp. 403-424.

Courtenay, S. and Davis, R. (2007). "Winter jet stream trends over the Northern Hemisphere". Quaterly Journal of Royal Meteorology Society, 133, pp. 2109-2115.

Egger, J. and Peter-Hoinka, K. (2008). " Mountain torques and synoptic systems in the Mediterranean”. Quaterly Journal of Royal Meteorology Society, 134, pp. 1067-1081.

Feldstein, S. B. (2007), "The dynamics of the North Atlantic Oscillation during the summer season". Quaterly Journal of Royal Meteorology Society, 133, pp. 1589-1518.

Gulev, S. K., Zuleva, O. and Reva, Y. (2000). "Synoptic and subsynoptic variability in the North Atlantic as revealed by the Ocean Weather Station data". Tellus, vol. 52, pp. 323-339.

IPCC. (2007). Climate Change 2007: The Physical Science Basis. Contribution of Working Group I to the Fourth Assessment Report of the Intergovernmental Panel on Climate Change [Solomon, S., D. Qin, M. Manning, Z. Chen, M. Marquis, K.B. Averyt, M. Tignor and H.L. Miller (eds.)]. Cambridge University Press, Cambridge, United Kingdom and New York, NY, USA, 996 pp.

IPCC. (2013). Climate Change 2013: The Physical Science Basis. Contribution of Working Group I to the Fifth Assessment Report of the Intergovernmental Panel on Climate Change [Stocker, T.F., D. Qin, G.-K. Plattner, M. Tignor, S.K. Allen, J. Boschung, A. Nauels, Y. Xia, V. Bex and P.M. Midgley (eds.)]. Cambridge University Press, Cambridge, United Kingdom and New York, NY, USA, 1535 pp, doi:10.1017/CBO9781107415324.

Jansa Guardiola, J. M. (1951). "Previsión del tiempo en el Mediterráneo occidental". Revista de Geofísica, no 39 , pp. 234-256.

Jansa Guardiola, J. M. (1966). "La Meteorología en el Mediterráneo Occidental". Tercer Ciclo de Conferencias, I.N.M. 1964, pp. 1-35.

Kazmin, A, Zatsepin, A. G. and Kontoyianis, H. (2009). "Comparative analysis of the long-term variability of winter surface temperature in the Black and Aegeus Seas during 1982-2004 associated with the large scale atmospheric forcing". International Journal of Climatology, vol. 30, pp.1349-1359.

Kidson, J. W. (1985). "Index cycles in the Northern Hemisphere during the G.W.E.". Mon. Weather Rev., 113, pp. 607-623. 
Lejenas, H. and Okland, H. (1983). "Characteristics of Northern Hemisphere blocking as determined from a long-time series of observational data". Tellus, 35 , A, pp.350-362.

Marshall, J., Kushnir, Y., Battisti, B., Chang, P., Czaja, A., Dickson, R., Hurrell, J., McCartney, M., Saravanan, R. and Visbeck, M. (2002). "North Atlantic climate variability phenomena, impacts and mecanisms". International Journal of Climatology, vol. 21, pp. 1863-1898.

Martin Vide, J. y Fernandez Belmonte, D. (2001). "El índice NAO y la precipitación mensual en la España peninsular". Investigaciones Geográficas, Instituto Universitario de Geografía, Universidad de Alicante, 26, pp. 45-58.

Martin Vide, J. y López Bustins, J.A. (2006). “The Western Mediteranean Oscillation and rainfall in the Iberian Peninsula". International Journal of Climatology, 26, pp. 1455-1475.

Meteorologie Nationale Francaise, (1962). Notice d'information technique de la Météorologie nationale francaise.

Merle, J. et Tourre, Y. (1983). "Le bilan energétique à l'interface air-mer". La Météorologie, VI, 33, pp.51-68.

Namias, J. (1987). "Factors relating to the explosive North Atlantic cyclone of December 1986". Weather, 42, pp. 323-326.

Namias, J. (1989). “Anomalous climatological background of the storm of 15-16 October 1987”. Weather, 44, pp. 98-104.

NCAR, (2010). "Monthly Northern Hemisphere 72x19 Tropospheric Analyses, continuing from April 1947”. ds085.1, Research Data Archive, National Center for Atmospheric Research, Boulder, Colorado. (http://dss.ucar.edu).

Noussier, O., Ducrocq, V. and Ricard, C. (2008). "A numerical study of three catastrophic precipitations events over souther France". Quaterly Journal of Royal Meteorology Society, 134, pp. 111-130.

Quereda Sala, J. (1986). "Un aspect du regime thermique en Mediterranée Occidentale”. Met-mar, 131, pp.13-18.

Quereda Sala, J. (1989a). "Blocage et goutte froide sur le Levant espagnol”. La Météorologie, Societé Météorologique de France, VII, 27, pp.2-7.

Quereda Sala, J. (1989b). "Las interacciones atmósfera-océano en la climatología del Mediterráneo Occidental". Avenidas fluviales e inundaciones en la cuenca del Mediterráneo. Alicante, Caja de Ahorros del Mediterráneo e Instituto Universitario de Geografía de la Universidad de Alicante. Pp. 67-90.

Quereda Sala, J. (1990). “Intense Cyclones in the Spanish Mediterranean”. Weather, Royal Meteorological Society, 45, 7, pp.278-279.

Quereda Sala, J. y Montón Chiva, E. (1994). Las lluvias torrenciales en la Comunidad Valenciana. Interacciones atmósfera-mar. Co-lecció Universitària. Diputacció de Castelló, Castelló. 190 pp.

Quereda Sala, J. Montón Chiva, E y Escrig, J. (2011). “Teleconnections between the North Atlantic SST and Mediterranean rainfall". Tethys, Journal of Mediterranean Meteorology and Climatology, 8, 2011, ACAM, pp. 31-42. 
Ratier, A. (1986). “Temperature de la mer et analyse objective”. Met-mar, 130, pp.12-17.

Rayner, N. A., Brohan, P., Parker, D. E., Folland, C. K., Kennedy, J. J., Vanicek, M., Ansell, T. and Tett, S. F. B. (2006). "Improved analyses of changes and uncertainties in sea surface temperature measured in situ since the mid-nineteenth century: the HadSST2 data set". Journal of Climate, 19, (3) pp. 446-469 (http:// hadobs.metoffice.com/hadsst2/index.html).

Rodwell, M. J. and Folland, C. K. (2002). "Atlantic aire-sea interaction and seasonal predictability”. QJRMS , Quaterly Journal of Royal Meteorology Society, 128, pp. 1413-1443.

Stephenson, D., Pavan, V. and Bojarin, R. (2000). " Is the North Atlantic Oscillation a random walk?”. International Journal of Climatology, vol. 20, pp. 1-18.

Stringer, E. (1972). Foundations of Climatology, W. H. Freeman, San Francisco, 586 pp.

Trigo, R. M., Pozo-Vázquez, D., Osborn, T. J., Castro-Díez, Y., Gámiz-Fortis, S. and Esteban-Parra, $\mathrm{M}^{\mathrm{a}}$. J. (2004). "North Atlantic Oscillation influence on precipitation, river flow and water resources in the Iberian Peninsula". International Journal of Climatology, vol. 24, pp. 925-944.

Watanabe, M. and Kimoto, M. (2006). "Atmosphere-ocean coupling in the North Atlantic: a positive feedback". Quaterly Journal of Royal Meteorology Society, 126, pp. 3343-3369.

Visbeck, M. H., Hurrell, J. W., Polvani, L. and Cullen, H. M. (2001). "The North Atlantic Oscillation: Past, present and future". Proc. Nat. Acad. Sci. USA 98, 12876-12877.

Vonder Haar, T. H. and Oort, A. H. (1973). "New estimate of annual poleward energy transport by northern hemisphere oceans". J. of Phys. Oceanogr., 3, pp. 169-172.

Zeng-Zeng, HU and Zhaohua WU, (2004). "The intensification and shift of the annual North Atlantic Oscillation in a global warming scenario simulation". Tellus A, vol, 56,2, pp. 90-112. 